Supporting Information

\title{
Iron Oxide-Coated Dextran Nanoparticles with Efficient Renal Clearance for Musculoskeletal Magnetic Resonance Imaging
}

\author{
Ji-wook Kim, , ,\# Jiyong Cheong, 2, " Hayeon Cheong, ${ }^{1}$
}

Jun Geol Yoon, ${ }^{3}$ Joon-Yong Jung, ${ }^{4}$ Jae-Hyun Lee, ${ }^{2 *}$ and Tae-Hyun Shin ${ }^{1,5^{*}}$

${ }^{1}$ Inventera Pharmaceuticals Inc., Seoul 08507, Republic of Korea.

${ }^{2}$ Graduate Program of NanoBiomedical Engineering, Advanced Science Institute, Yonsei

University, Seoul 03722, Republic of Korea.

${ }^{3}$ Department of Clinical Korean Medicine, Graduate School, Kyung Hee University, Seoul

02447, Republic of Korea.

${ }^{4}$ Department of Radiology, Seoul St. Mary's Hospital, The Catholic University of Korea,

Seoul 06591, Republic of Korea.

${ }^{5}$ Institute of Radiological Science, Yonsei University, Seoul 03722, Republic of Korea. 
\#J.-w.K. and J.C. contributed equally to this work.

Correspondence should be addressed to J.-H.L. and T.-H.S.

E-mail: jhyun_lee@yonsei.ac.kr and thshin87@yuhs.ac 


\section{Materials and methods}

\section{Materials}

Dextran T10 (molecular weight, 10,000 g/mol) was purchased from Pharmacosmos. All other chemicals were obtained from Sigma-Aldrich and used as received unless otherwise specified.

\section{Synthesis of i-IONPs and USPIO}

i-IONPs were synthesized following slight modification of reported procedure. ${ }^{1}$ Briefly, $20 \mathrm{mM}$ Dextran T10 (5510 0010 9001, Pharmacosmos) solution was mixed with sodium hydroxide solution and $75 \mathrm{mmol}$ epichlorohydrin (45340, Sigma Aldrich) followed by dropwise addition of $380 \mathrm{mmol}$ of ethylenediamine (E26266, Sigma-Aldrich). The cross-linked dextran was purified via $10 \mathrm{kDa}$ molecular weight cut-off ultrafiltration filter (UFC9010, Millipore). Then, succinic anhydride (239690, Sigma-Aldrich) was added to the cross-linked dextran solution and incubated for $24 \mathrm{~h}$. After ultrafiltration, carboxyl-functionalized dextran was reacted with iron chloride solution under basic $\mathrm{pH}$ for $1 \mathrm{~h}$. A more excess amount of iron chloride was reacted for elemental and crsyatalline structure analyses. The final products were purified and concentrated using ultrafiltration. Ultrasmall superparamagnetic iron oxide nanoparticle (USPIO) was synthesized according to a previous report. ${ }^{2,3} 10 \mathrm{mmol}$ of iron-oleate complex, $60 \mathrm{mmol}$ of oleyl alcohol (A18018, Alfa Aesar), and 294 mmol of diphenyl ether were added to a $250 \mathrm{~mL}$ round bottom flask. The mixture was heated to $200^{\circ} \mathrm{C}$ at a constant heating rate of $10^{\circ} \mathrm{C} / \mathrm{min}$ and then cooled to RT. Synthesized nanoparticles were precipitated with an excess amount of acetone and then redispersed to tetrahydrofuran (186562, Sigma-Aldrich). Four milliliters of iron oxide nanoparticle-tetrahydrofuran solution $(10 \mathrm{mg} / \mathrm{mL})$ was mixed with $400 \mathrm{mg}$ of PEG-phosphate in ethanol and heated at $70{ }^{\circ} \mathrm{C}$ for $8 \mathrm{~h}$. The final product was purified by a dynamic dialysis device with a $20 \mathrm{kDa}$ molecular weight cutoff (MWCO; 137048, Interchim).

\section{TEM analyses}

TEM analysis was conducted by using the JEM-2100 Plus (JEOL). i-IONPs were negatively stained using freshly prepared uranyl formate aqueous solution $(0.75 \mathrm{wt} \%)$. Cryo-TEM analysis was carried out by using the Tecnai G2 Spirit TWIN (FEI) equipped with cryo transfer holder. For elemental mapping, the JEM-ARM200F (JEOL) equipped with a cold field emission gun, an electron energy loss spectroscopy detector (Model 965 GIF Quantum ER/S; Gatan) and a charge-coupled device camera (OneView 16M pixels; Gatan) was utilized. Samples were investigated at an acceleration voltage of $80 \mathrm{kV}$.

\section{$\underline{\text { Hydrodynamic size and zeta potential measurement }}$}

The hydrodynamic size and zeta potential were measured using a dynamic light scattering (DLS, Zetasizer Pro, Malvern Panlaytical) device with the following parameters: reflective index of 1.48 and absorption of 0.001 . 


\section{Colloidal stability test}

i-IONPs were incubated in $1 \mathrm{X}$ phosphate buffered saline $(\mathrm{pH} 7.4, \mathrm{NaCl} 150 \mathrm{mM})$ and their hydrodynamic size was measured using DLS device. USPIO was mixed with iodinated contrast agent (Iopamidol 300, Bracco Imaging) to create $10 \%$ and $50 \%$ Iopamidol solutions.

\section{Metal leaching stability test}

i-IONPs were incubated in blood plasma (H3667, Sigma-Aldrich) and filtered using an Amicon unit (3 kDa MWCO, Merck) five times. After the supernatant was collected, the concentration of iron and dextran was measured using respective ICP-MS (7900, Agilent) and phenol-sulfuric acid method.

\section{Gel permeation chromatography (GPC)}

Molecular weight of i-IONPs was measured by size exclusion liquid chromatography using hydroxylated methacrylate phase column (TSKgel ${ }^{\circledR} \mathrm{GMPW}_{\mathrm{XL}}$, Tosoh) and $0.1 \mathrm{M} \mathrm{NaNO}_{3}$ as a moving phase. To have the relationship between retention time and hydrodynamic size, size exclusion column was calibrated using dextran standards with nine different molecular weights $(0.181,0.667,6.1,9.6,21.1,47.1,107,337$, and $642 \mathrm{kDa})$.

\section{Thermogravimetric analysis (TGA)}

TGA was performed on a thermogravimetric analyzer (TGA Q500, TA Instruments). Temperature range was set from ambient to $900^{\circ} \mathrm{C}$ with a temperature ramp of $10^{\circ} \mathrm{C} \mathrm{min}^{-1}$. Thermal decomposition was recorded under $\mathrm{N}_{2}$ gas.

\section{Fourier-transform infrared spectroscopy (FTIR)}

i-IONPs were analyzed with attenuated total reflectance (ATR)-FTIR spectrometer (FT-IR 4600, Jasco). The scanning range was set from 4000 to $500 \mathrm{~cm}^{-1}$ with resolution of $4 \mathrm{~cm}^{-1}$ and 32 scans per sample.

\section{$\underline{\text { X-ray photoelectron spectroscopy (XPS) }}$}

XPS analysis was performed on a K-alpha (Thermo Scientific Inc., U.K.) with beam size of $400 \mu \mathrm{m}$ and beam power of $72 \mathrm{~W}(12,000 \mathrm{~V}$ and $6 \mathrm{~mA})$. The position of the $\mathrm{C} 1 \mathrm{~s}$ peak was taken as a reference for charge correction, with a banding energy of $285 \mathrm{eV}$.

\section{$\underline{\text { X-ray diffraction (XRD) measurement }}$}

XRD was conducted using a MiniFlex benchtop X-ray diffractometer (Rigaku) with a 2D hybrid pixel array detector (HyPix-400 MF; Rigaku). Samples were lyophilized and ground to form a powder. 


\section{Magnetization measurement}

Magnetization of each aqueous solution of i-IONPs, Dotarem, and USPIO was measured using a vibrating sample magnetometer (Lake Shore Cryotronics, Inc.). The mass of Fe or Gd of each sample was measured using inductively coupled plasma optical emission spectroscopy (ICP-OES) for mass magnetization.

\section{MRI measurement}

MRI systems and pulse sequences utilized in this study are as follows. (i) A 3 T preclinical MRI system (MRS 3017 model, MR Solutions, Inc.) with the following specifications: clear-bore size, $17 \mathrm{~cm}$; gradient strength, $600 \mathrm{mT} / \mathrm{m}$; RF amplifier power, $500 \mathrm{~W}$; RF coil, rat body volume coil; and operating software, preclinical scan. (ii) A 9.4 T preclinical MRI system (Biospec 94/20 USR, Bruker BioSpin) with the following specifications: clear-bore size, $20 \mathrm{~cm}$; gradient strength, $660 \mathrm{mT} / \mathrm{m}$; RF amplifier power, 1,000 W; RF coil, rat body volume coil and 4-channel surface coil. For the $T_{1}$ value measurements, an inversion-recovery-weighted fast spin-echo sequence with the following parameters was utilized: inversion time (TI), 50, 150, 300, 600, 1,000, 1,500, 2,500, and 5,000 ms; TR, 10,000 ms; TE, $11 \mathrm{~ms}$; field of view (FOV), $50 \times 50 \mathrm{~mm}^{2}$; slice thickness, $2 \mathrm{~mm}$; matrix size, $128 \times 128$; and echo train length (ETL). For the $T_{2}$ value measurements, a multi-echo spin-echo sequence was utilized; the sequence had the following parameters: TR, 5,000 ms; TE, $10-1,280 \mathrm{~ms}$; FOV, $50 \times 50 \mathrm{~mm}^{2}$; slice thickness, $2 \mathrm{~mm}$; and data matrix, $128 \times 128$.

\section{Cytotoxicity test}

Cytotoxicity of i-IONPs was assessed using human embryonic kidney cell line (HEK-293) and human vein endothelial cell line (HUVEC) by cell counting kit-8 (CCK-8) assay according to the protocol provided by the manufacturer (ab228554, Abcam). Varying concentrations of i-IONPs $(20,50,100,250,500,1,000[\mathrm{Fe}] \mathrm{mg} / \mathrm{mL})$ were treated.

\section{$\underline{\text { Animal injection }}$}

Sprague-Dawley (SD) rats (Orient Bio) were used in this study. All experiments were conducted under protocols approved by the Institutional Animal Care and Use Committee of Yonsei University College of Medicine (approval numbers: 2019-0284). For MRI scanning, rats were anesthetized with isoflurane (Hana Pharm. Co. Ltd.) in oxygen. The contrast agent was intra-articularly injected.

\section{Histological analysis}

The organs were fixed in 10\% neutral buffered formalin and then processed for Perls' blue iron-staining and hematoxylin and eosin (H\&E) staining according to the protocol provided by the manufacturer (ab150674 and ab245880, Abcam). The images were examined under a digital microscope. 


\section{Urine analysis}

i-IONPs were intra-articularly administered to the knee joint of male $(n=5)$ rats. For comparison, the same amount of saline was injected into the knee joint. Rats were housed in cages to allow for the collection of urine. The iron content in urine collected at $0 \sim 24,24 \sim 48,48 \sim 72 \mathrm{~h}$ was analyzed using inductively coupled plasma mass spectrometry (ICP-MS, 7900, Agilent). The dextran content in urine was analyzed using the phenolsulfuric acid method.

\section{Blood chemistry}

Blood samples were obtained from the abdominal aorta and centrifuged for serum separation. The blood urea nitrogen (BUN), serum creatinine (SCr), alanine transaminase (ALT), aspartate transaminase (AST), alkaline phosphatase (ALP), and gamma-glutamyl transferase (GGT) levels were analyzed using a biochemistry automatic analyzer (7180, Hitachi).

\section{Biodistribution study}

For the biodistribution study, rats were anesthetized and dose of i-IONPs at $124 \mu \mathrm{mol}[\mathrm{Fe}] / \mathrm{kg}(\mathrm{n}=4)$ was injected through tail vein. Rats were sacrificed followed by perfusion and collection of organs including brain, heart, liver, spleen, lungs, and tail. Isolated organs were washed with normal saline and lyophilized. The iron content of each organ was analyzed using inductively coupled plasma mass spectrometry (ICP-MS, 7900, Agilent).

\section{Statistical analysis}

Data are presented as the means \pm standard deviation of experiments repeated at least three times unless specified. Where appropriate, a Student's t-test was used $\left(* \mathrm{P}<0.05, * * \mathrm{P}<0.01,{ }^{*} * * \mathrm{P}<0.001\right)$. 


\section{Supplementary figures and table}

a

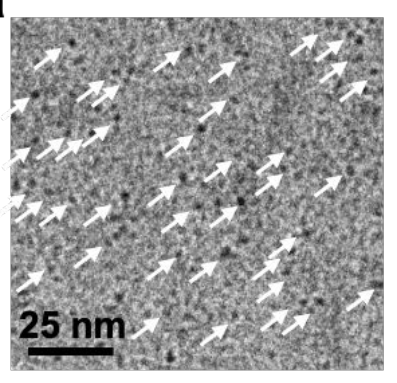

b

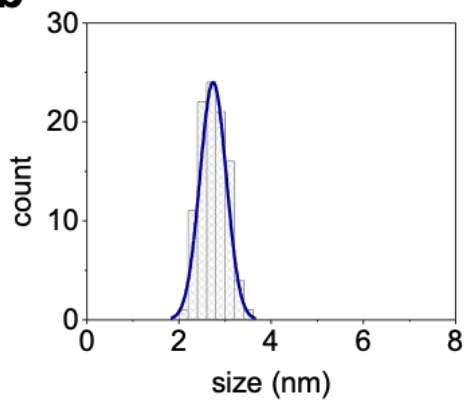

\section{C}
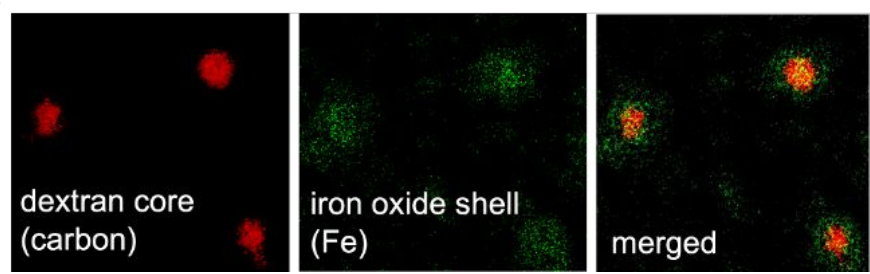

Figure S1. TEM analysis of i-IONPs. (a) Cryo-TEM image of i-IONPs. (b) Size distribution of i-IONPs analyzed from TEM image. (c) Elelemtal carbon (red) and iron (green) map of i-IONPs and their merged image.

a

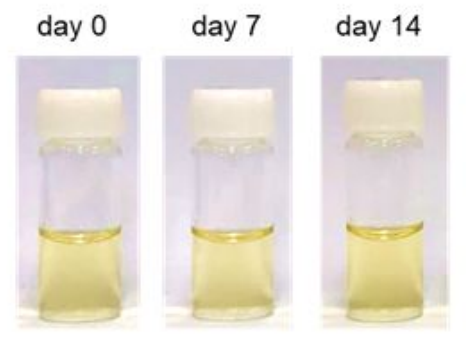

b

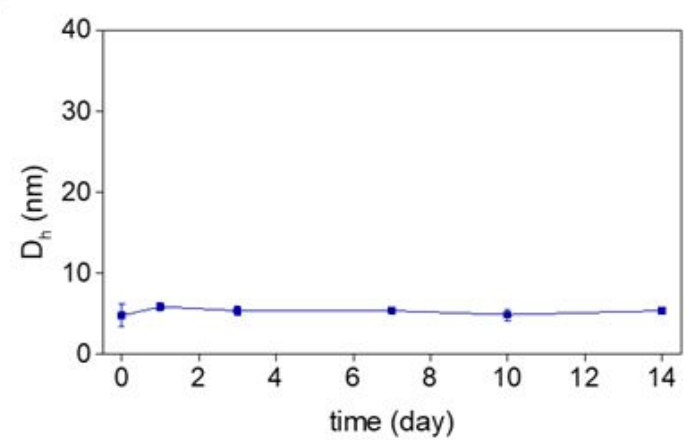

Figure S2. Colloidal stability of i-IONPs. (a) Photos and (b) $D_{h}$ of i-IONPs in physiological buffer solution (150 mM NaCl, pH 7.4). 

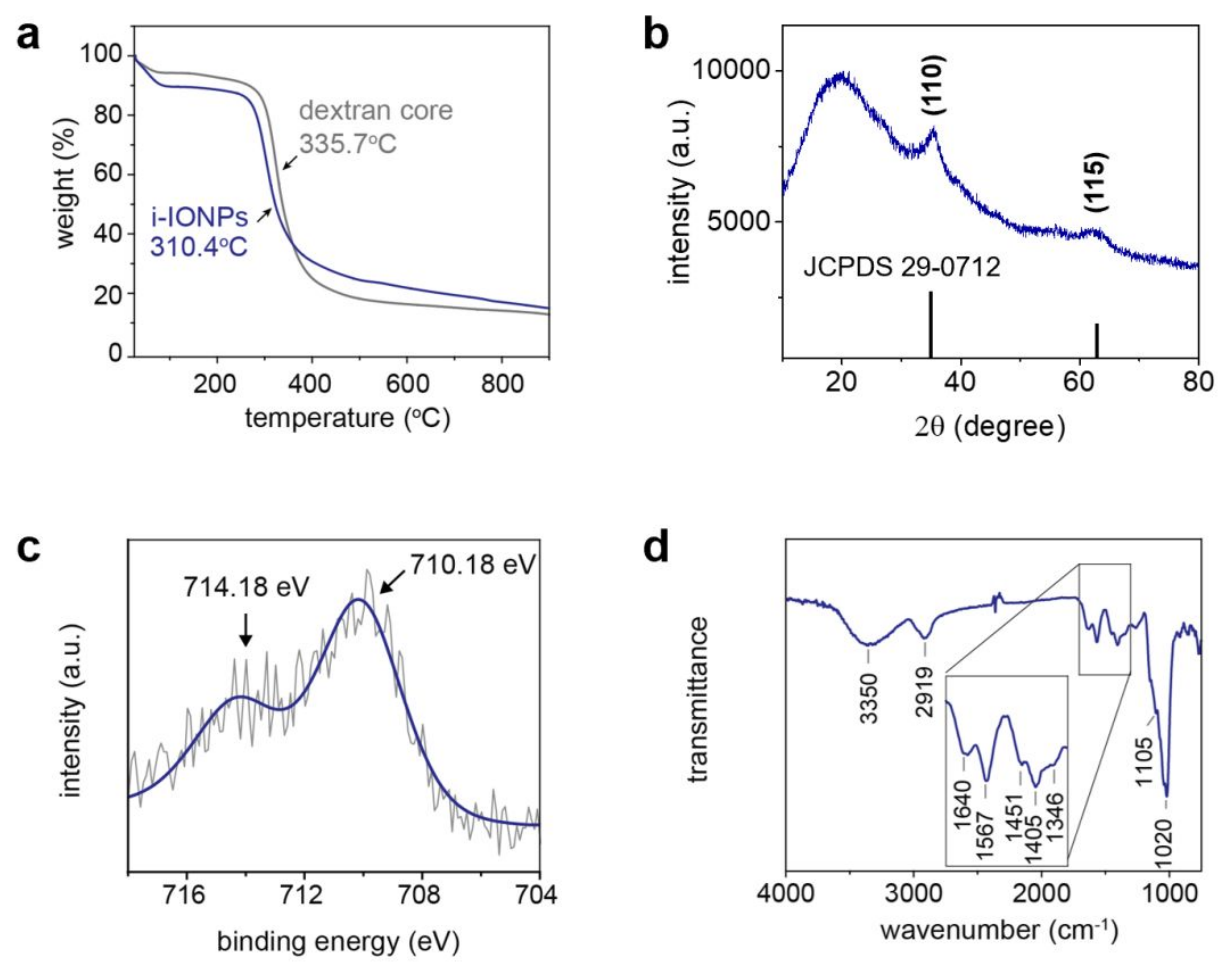

Figure S3. Physicochemical characterizations of i-IONPs. (a) TGA analysis of i-IONPs (blue) and dextran core (grey). (b) XRD pattern of i-IONPs. The diffraction peaks were indexed as 2-line ferrihydrite (Joint Committee on Powder Diffraction Standards, JCPDS, card number 29-0712). (c) XPS spectrum of i-IONPs. (d) FTIR spectrum of i-IONPs.
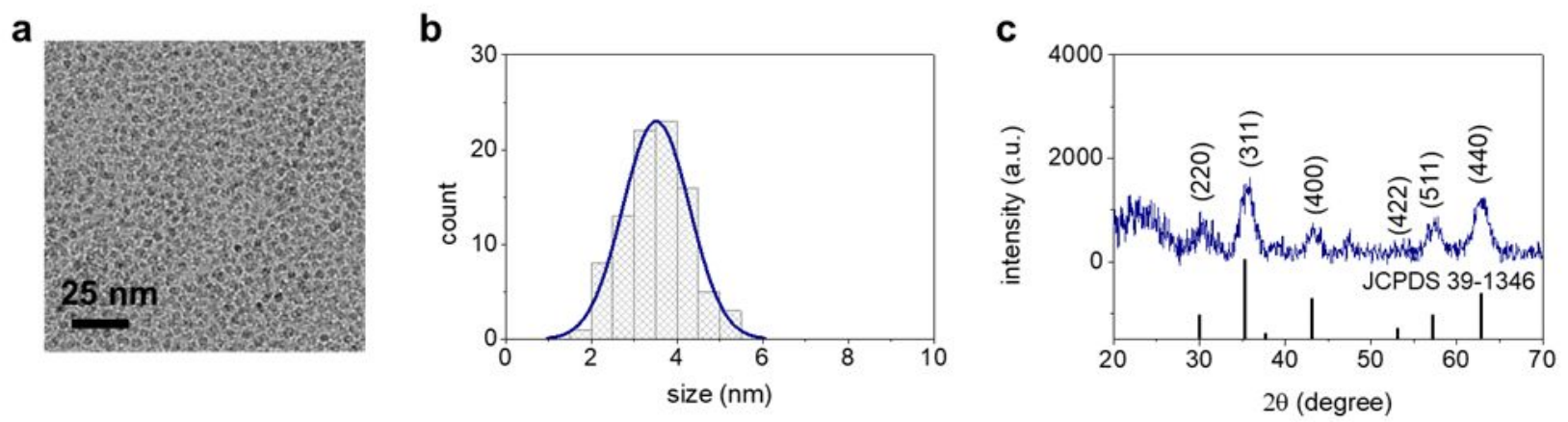

Figure S4. Characterization of USPIO. (a) TEM image and (b) size distribution of USPIO. (c) XRD pattern of USPIO. All peaks were indexed as crystalline maghemite (JCPDS card number 39-1346). 
a

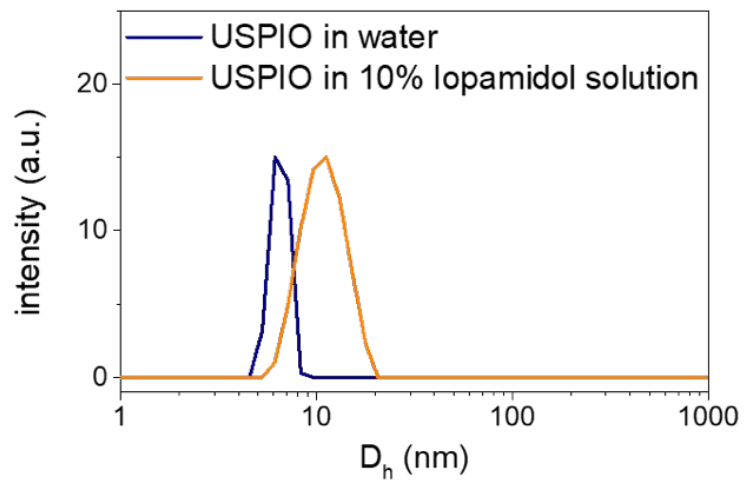

b

USPIO in water

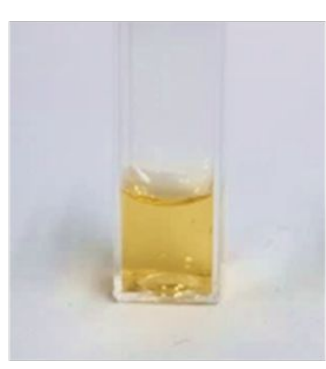

USPIO in $50 \%$ lopamidol solution

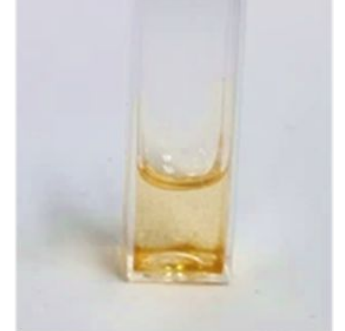

Figure S5. Colloidal stability of USPIO in Iopamidol solution. (a) $D_{h}$ of USPIO dispersed in water (blue) and in 10\% Iopamidol solution (orange). (b) Photo of USPIO dispersed in water (left panel) and in 50\% Iopamidol solution (right panel).

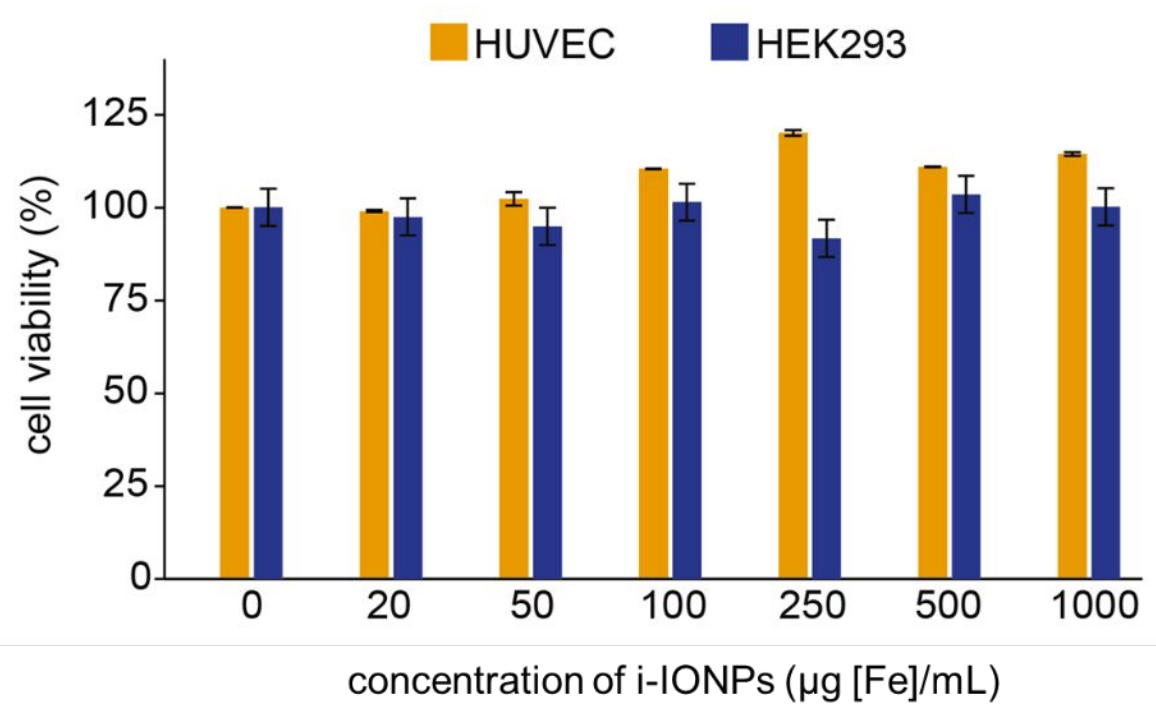

Figure S6. Cytotoxicity test of i-IONPs. The viability of HUVEC (orange) and HEK 293 (blue) assessed by CCK-8 assay after treating various concentrations of i-IONPs $(20,50,100,250,500$, and 1,000 $\mu \mathrm{g}[\mathrm{Fe}] / \mathrm{mL})$ 

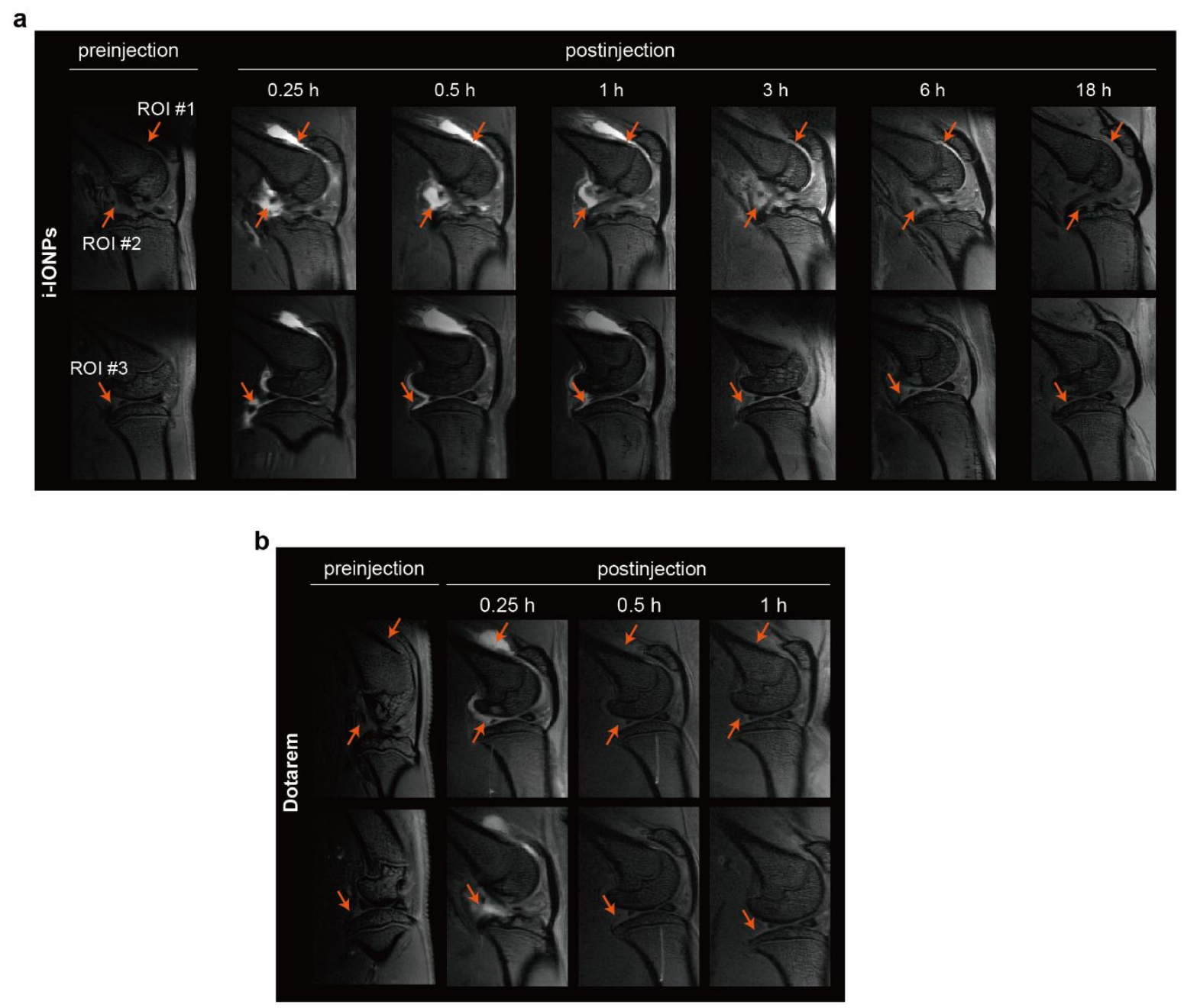

Figure S7. Comparison of the temporal imaging windows of i-IONPs and Dotarem. (a) $T_{1}$-weighted MR images scanned at different time points before and $0.25 \mathrm{~h}, 0.5 \mathrm{~h}, 1 \mathrm{~h}, 3 \mathrm{~h}, 6 \mathrm{~h}$, and $18 \mathrm{~h}$ after injection of i-IONPs. (b) $T_{1}$-weighted MR images were obtained before and $0.25 \mathrm{~h}, 0.5 \mathrm{~h}$, and $1 \mathrm{~h}$ after injection of Dotarem. SNR is measured from three different regions of interest marked in MR images as orange arrows. 

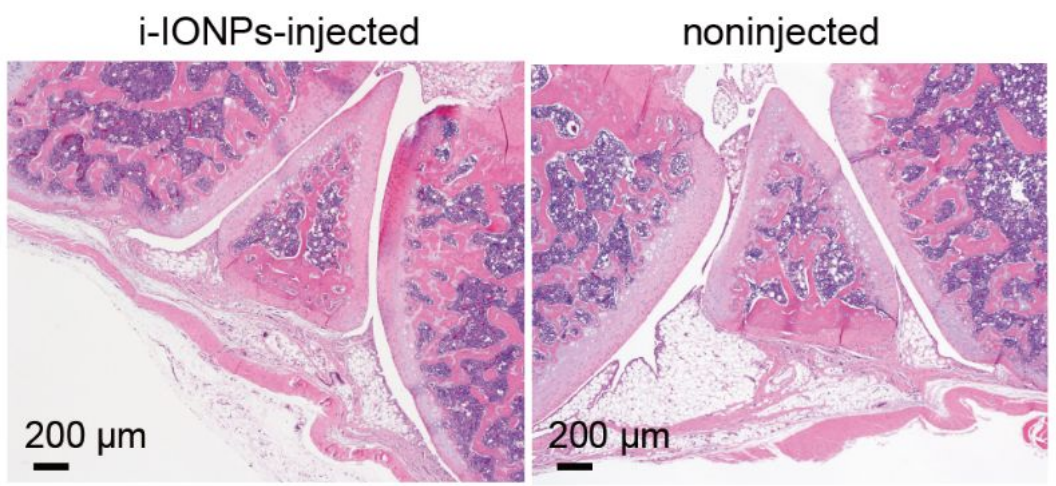

Figure S8. Representative H\&E-stained histological images of rat joints. left, i-IONPs-injected group (injection dose $=2.9 \mu \mathrm{mol}[\mathrm{Fe}] /$ head); right, noninjected control group.
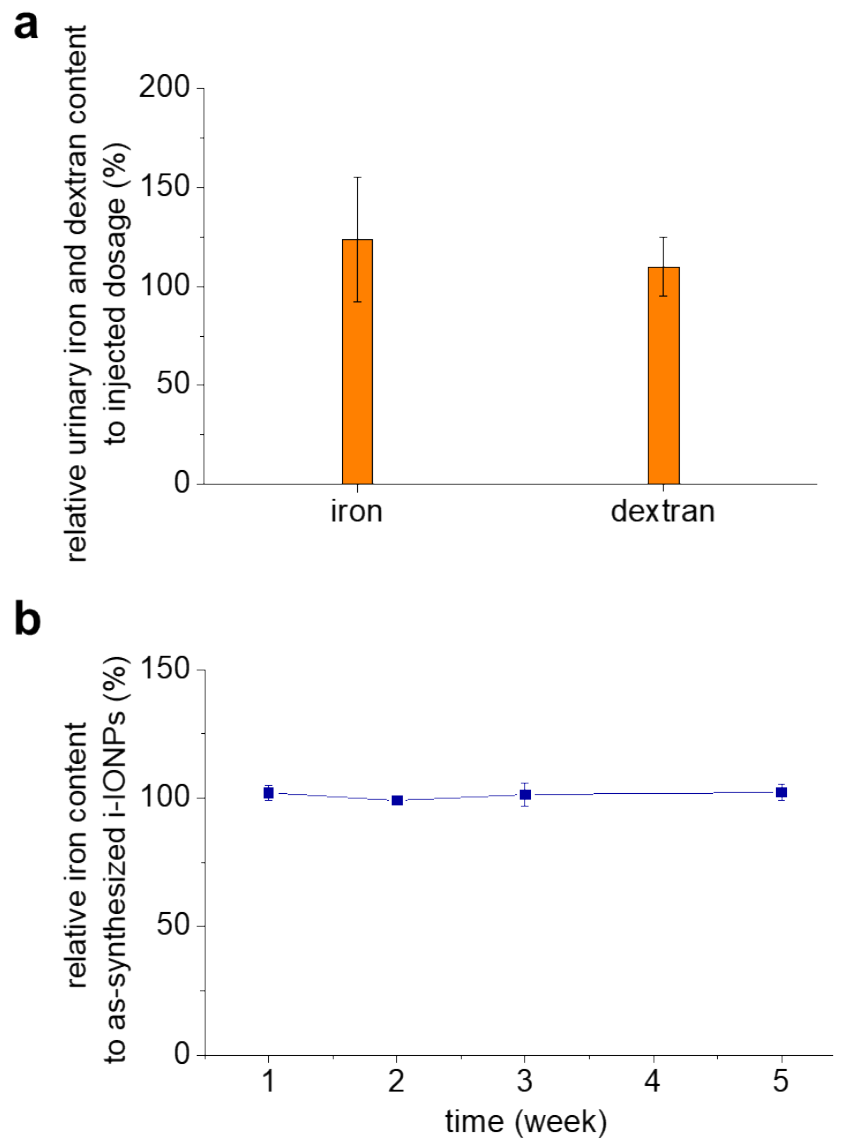

Figure S9. Metal leaching stability of i-IONPs in urine and blood plasma. (a) The relative urinary content of iron and dextran to that of injection dosage. Urine samples were collected from five biologically independent male rats injected with i-IONPs. (b) Stability of i-IONPs in blood plasma. Relative iron content compared to assynthesized i-IONPs is plotted against time. 


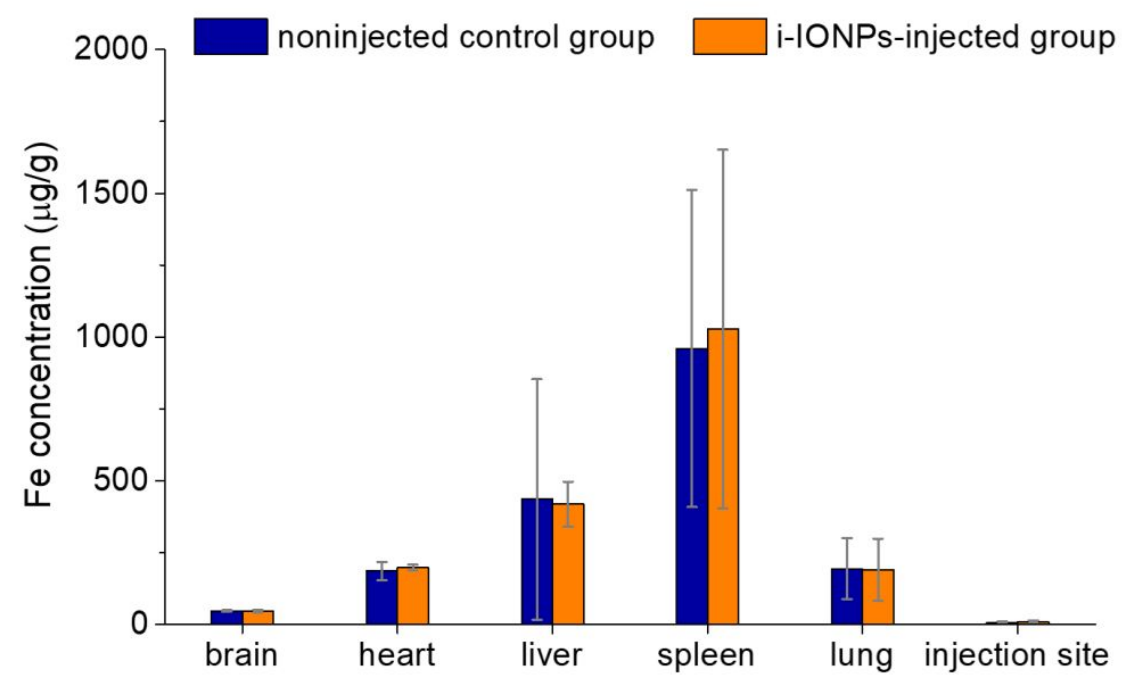

Figure S10. Biodistribution of i-IONPs. Iron concentration at the brain, heart, liver, spleen, lungs, and injection site (tail) of noninjected control group (blue) and i-IONPs-injected group (orange, injection dose $=124 \mu \mathrm{mol}$ $[\mathrm{Fe}] / \mathrm{kg}, \mathrm{n}=4$ biologically independent male rats for each group).

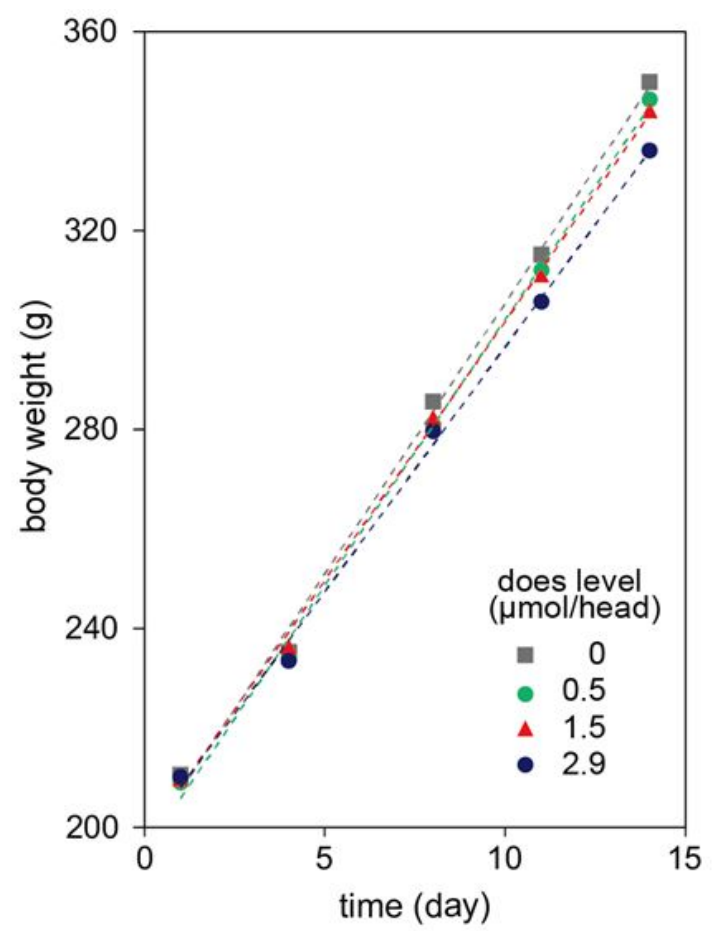

Figure S11. Rat body weight test after the intra-articular injection of i-IONPs (injection dose $=0.5,1.5$, and 2.9 $\mu \mathrm{mol}[\mathrm{Fe}] /$ head, $\mathrm{n}=5$ biologically independent male rats for each group). 
Table S1. Blood chemistry test of i-IONPs.

\begin{tabular}{|c|c|c|c|c|c|c|}
\hline $\begin{array}{c}\text { Dose } \\
(\mu \mathrm{mol}[\mathrm{Fe}] \\
/ \mathrm{head})\end{array}$ & $\begin{array}{c}\text { BUN } \\
(\mathrm{mg} / \mathrm{dL})\end{array}$ & $\begin{array}{c}\mathrm{SCr} \\
(\mathrm{mg} / \mathrm{dL})\end{array}$ & $\begin{array}{c}\text { ALT } \\
(\mathrm{U} / \mathrm{L})\end{array}$ & $\begin{array}{c}\text { AST } \\
(\mathrm{U} / \mathrm{L})\end{array}$ & $\begin{array}{c}\text { ALP } \\
(\mathrm{U} / \mathrm{L})\end{array}$ & $\begin{array}{c}\text { GGT } \\
(\mathrm{U} / \mathrm{L})\end{array}$ \\
\hline 0 & $11.4 \pm 0.7$ & $0.42 \pm 0.04$ & $32.9 \pm 2.9$ & $68.1 \pm 5.5$ & $892.0 \pm 149.2$ & $0.34 \pm 0.08$ \\
\hline 0.5 & $10.4 \pm 1.4$ & $0.41 \pm 0.03$ & $31.2 \pm 5.1$ & $78.5 \pm 15.3$ & $701.2 \pm 180.1$ & $0.33 \pm 0.09$ \\
\hline 1.5 & $10.6 \pm 1.8$ & $0.41 \pm 0.02$ & $27.5 \pm 2.5$ & $76.0 \pm 11.8$ & $691.5 \pm 133.8$ & $0.46 \pm 0.07$ \\
\hline 2.9 & $12.4 \pm 1.5$ & $0.45 \pm 0.03$ & $31.0 \pm 1.8$ & $72.1 \pm 8.6$ & $794.2 \pm 185.7$ & $0.26 \pm 0.07$ \\
\hline
\end{tabular}

\section{References}

1 Shin, T. H.; Kim, P. K.; Kang, S.; Cheong, J.; Kim, S.; Lim, Y.; Shin, W.; Jung, J. Y.; Lah, J. D.; Choi, B. W.; Cheon, J. High-Resolution $T_{1}$ MRI via Renally Clearable Dextran Nanoparticles with an Iron Oxide Shell. Nat. Biomed. Eng. 2021, 5, 252-263.

2 Kim, B. H.; Lee, N.; Kim, H.; An, K.; Park, Y. Il; Choi, Y.; Shin, K.; Lee, Y.; Kwon, S. G.; Na, H. Bin; Park, J.; Ahn, T.; Kim, Y.; Moon, W. K.; Choi, S. H.; Hyeon, T. Large-Scale Synthesis of Uniform and Extremely Small-Sized Iron Oxide Nanoparticles for High-Resolution $\mathrm{T}_{1}$ Magnetic Resonance Imaging Contrast Agents. J. Am. Chem. Soc. 2011, 133, 12624-12631.

3 Wei, H.; Bruns, O. T.; Kaul, M. G.; Hansen, E. C.; Barch, M.; Wiśniowska, A.; Chen, O.; Chen, Y.; Li, N.; Okada, S.; Cordero, J. M.; Heine, M.; Farrar, C. T.; Montana, D. M.; Adam, G.; Ittrich, H.; Jasanoff, A.; Nielsen, P.; Bawendi, M. G. Exceedingly Small Iron Oxide Nanoparticles as Positive MRI Contrast Agents. Proc. Natl. Acad. Sci. U. S. A. 2017, 114, 2325-2330. 